\title{
Necrotizing Fasciitis and Sepsis from Group A Streptococcus Following Cesarean Section
}

\author{
Daiva Bartkeviciene*, Ruta Kurtinaitiene, Mindaugas Silkunas, Gediminas Januska and Vilius \\ Rudaitis
}

Vilnius University Hospital, NHS Clinics, Vilnius, Lithuania

*Corresponding author: Daiva Bartkeviciene, Associate Professor at Vilnius University Hospital, NHS Clinics, Vilnius, Lithuania, Tel: +37068725702; E-mail: daivabartk@gmail.com

Received date: 26 December, 2016; Accepted date: 20 February, 2017; Published date: 22 February, 2017

Citation: Bartkeviciene D, Kurtinaitiene R, Silkunas M, et al. Necrotizing Fasciitis and Sepsis from Group A Streptococcus Following Cesarean Section, Gynecol Obstet Case Rep. 2017, 3:1.

\section{Introduction}

Necrotizing fasciitis (NF) is a rare superficial fascia and subcutaneous tissue infection which may take a lifethreatening course with systemic toxicity and a high mortality rate which can exceed $5 \%$ to $12 \%[1-3]$.

Necrotizing fasciitis can manifest as two types. Type I is a synergistic polymicrobial infection of both aerobes and anaerobes (mostly, S. aureus, Streptococci, Enterocci, E. coli, B. fragilis, Clostridia) most common in patients with diabetes, peripheral vascular disease and after operations. Type II is a monomicrobial infection of group A streptococcus (Streptococcus pyogenes) and less frequently methicillinresistant Staphylococcus aureus (MRSA) seen in patients without underlying comorbidities [4]. Group A Streptococcus (GAS) postpartum infections remain the most common cause of severe maternal postpartum infections and death worldwide $[5,6]$. The scenario in the obstetric population is further complicated by associated comorbidities such as diabetes mellitus, malnutrition and anaemia [1,4]. However, the single most important risk factor for postpartum maternal infection is Caesarean section $[7,8]$.

The signs and symptoms of GAS necrotizing fasciitis can be divided into early and later manifestations. The early presenting signs include localized edema, induration, exquisite pain at the site of infection and erythema [4,9]. Non-specific symptoms and signs of infection include fever, arthralgia and myalgia. If not recognized and treated, systemic toxicity, wound anesthesia and progression to frank necrosis may ensue over hours to days $[4,9]$. The infection can spread up to $2.5 \mathrm{~cm} / \mathrm{h}$ with minimal change in the overlying skin [10].

Early diagnosis and treatment lead to decreased mortality. Unfortunately, no single non-invasive test is adequate for diagnosis. Surgical exploration and biopsy remain only diagnostic methods able to rule out necrotizing fasciitis definitively and should not be delayed for imaging [4,9]. In addition to surgical diagnosis, blood cultures are often taken but have limited utility $[4,9]$.

Treatment principles for necrotizing fasciitis remains early aggressive surgical debridement with broad spectrum antibiotic coverage $[4,9,11,12]$. Optimal surgical treatment is achieved by removing all necrotic tissue to a bleeding edge. Incisions are often left open to facilitate daily re-exploration of the wound with frequent wound changes and additional debridement as warranted [4]. It is founded that mortality rate can exceed $75 \%$ when surgical treatment is delayed by $\geq 48$ hours compared to $12 \%$ mortality in those who were treated in $\geq 12$ hours [11]. As with all cases of severe sepsis and septic shock, early and aggressive resuscitation improve outcomes. Hypotension and shock must be rapidly reversed, and often several litres of intravenous (IV) fluid as well as vasopressor therapy are required [9].

\section{Case Report}

A 21 year-old Caucasian woman was presented with fever, mild abdominal pain and strong weakness with suspicion of abdominal wall hematoma. Five days ago she gave birth to a healthy, full-term infant by Caesarean section. She was discharged from hospital on postpartum day 3 after an uneventful postoperative recovery. The patient denied any past medical or surgical history. It was second delivery in a year.

Objective findings on arrival to emergency room: the patient had a fever of $39.0^{\circ} \mathrm{C}$, a heart rate of $110 \mathrm{bpm}$, a blood pressure of $90 / 60 \mathrm{mmHg}$ and a respiratory rate of 16 breaths per minute. During examination redness, infiltration, painful palpation and blistering rashes of anterior abdominal wall were observed. Serous-hemorrhagic vaginal discharge, elevated, soft and painless uterus with non-pathological parametrium was discovered during vaginal examination. Vaginal ultrasound revealed $10 \times 9 \times 8 \mathrm{~cm}$ uterus, normal endometrial cavity and small amount of pelvic fluid. The laboratory findings included total leucocyte count $21,800 / \mu \mathrm{L}$, platelet count $89,000 / \mathrm{ml}$, C-reactive protein $317 \mathrm{mg} / \mathrm{l}$, procalcitonin 15,98 $\mu \mathrm{g} / \mathrm{L}$, total protein level 4,44 g/dL, serum albumin level $2.06 \mathrm{~g} / \mathrm{dL}$. Abdominal ultrasound showed that subcutaneous and muscle layers of anterior abdominal wall below the navel were markedly thickened and swollen. According to physical examination, laboratory and instrumental evaluation, initial empiric antibiotic therapy was started with Piperacillin/Tazobactam $4.5 \mathrm{~g}$ and $0.5 \mathrm{~g}$ 
Metronidazole. Patient's condition was deteriorating, because of fever rising to $40^{\circ} \mathrm{C}$ with chills, intoxication, hemodynamic instability patient was transferred from Gynecology department to intensive care unit.

Five hours after hospitalization laparotomy was performed. Revision of CS wound scar, aponeurosis, pelvic and abdominal organs and drainage of peritoneal cavity and wound were made. During the operation, about $70 \mathrm{ml}$ purulent, malodorous fluid with fibrin plaque in subcutaneous layer, regions of aponeurosis, straight abdominal muscles, minor pelvis and near the left parametre was founded. Uterus was in normal consistency with whitish fibrin crusty on Caesarean section scar. Streptococus beta-haemolyticus gr. A and Bacteroides vulgatus were recovered from the specimens of subcutaneous layers and aponeurosis region. Her laboratory results showed leucocitopenia, thrombocytopenia, elevated Creactive protein and procalcitonin values. Patient's fluid balance reached $+3587 \mathrm{ml}$ (weight for woman-55 kg) and intestinal obstruction was observed.

The patient's condition remained serious and septic with hemodynamic instability. According to microbiology culture sensitivities antibiotic therapy was changed to Meropenem 2 gms, Vancomycin $1 \mathrm{~g}$ and Metronidazole $0.5 \mathrm{~g}$ intravenously. Hypovolemia correction with fluid infusion, anticoagulant therapy, blood pressure maintaining with vasopressors infusion was continued constantly. 24 hours after hospitalization total hysterectomy with additional abdominal wall incisions was performed together with wound tamponade and abdominal cavity drainage (Figure 1 ).

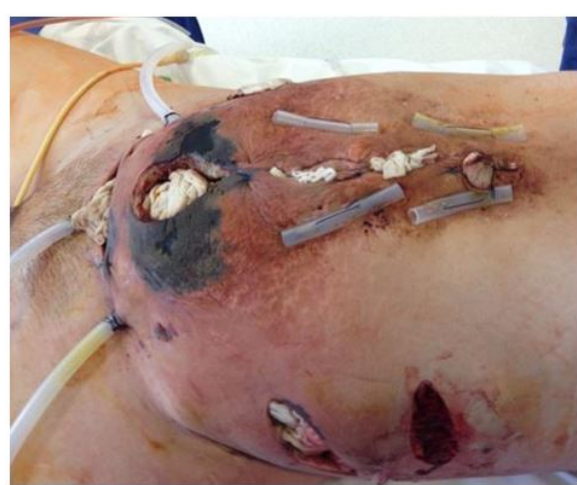

Figure 1 Objective findings before second operation.

Bacterial culture test from abdominal wall wound was repeated which showed the increase growth of Streptococus beta-haemolyticus gr. A. 25 hours after the second operation, the patient had episode of generalized tonic-clonic seizures. CT scan of the brain showed edema, MRI of the brain-expressed brain and cerebellum edema, bright intracranial hypertension. According to the patient's condition worsening, antibiotic therapy was changed to Penicillin 4 million units, Meropenem $2 \mathrm{~g}$ and $0.5 \mathrm{~g}$ of Metronidazole per day intravenously. Brain CT the next day showed progressive intracranial hypertension and cerebellar tonsillar descent below foramen magnum. Four days after patient's presentation to the hospital, secondary acute meningoencephalitis, cerebral edema, brain stem and cerebellum dislocation and herniation was diagnosed. 6 days after hospitalization the patient died.

\section{Discussion}

A case of necrotizing fasciitis and sepsis from Group A Streptococcus following Caesarean section is discussed.

NF is relatively rare, fulminating soft-tissue infection. Mostly, it starts following trauma and, rarely, a surgical procedure [1]. Various predisposing factors, such as diabetes, obesity, malnutrition, cancer, alcoholism, immunosuppression, peripheral vascular disease, advanced age are described for NF [1]. A minor penetrating injury or a surgical incision is usually involved, with postoperative cases accounting for approximately $20 \%$ to $50 \%$ of the total $[4,13]$. In our case Caesarean section, short time between pregnancies, low serum total protein and serum albumin values, undernourishment, were found to be associated with NF. The etiologic isolates consist of Gram-positive organisms, such as Staphylococcus aureus, S. pyogenes, and Enterococci; Gramnegative aerobes, such as Escherichia coli and Pseudomonas species; and anaerobic organisms, such as Bahides or Clostridium species $[4,14,15]$. In this case both GAS and Bacteroides were presented. Regardless of delivery type, postpartum patients have a 20 -fold increased incidence of GAS-induced postpartum infection compared to non-pregnant women [16]. Lamagni et al. founded that S. pyogenes causes $3 \%$ of all puerperal sepsis cases [17]. The mechanisms behind GAS bacterial virulence, postpartum susceptibility and the immune response to female reproductive tract infections remain poorly understood [8].

Puerperal infections, including NF, following GAS present rapidly, within 2 to 48 hours postpartum and can be nonspecific, delaying treatment [8]. As in our case, initial presentation of NF is often mistaken for cellulitis or wound hematoma [1]. Approximately half the patients with GAS necrotizing fasciitis have concomitant streptococcal toxic shock syndrome (STSS) [9]. This is characterized by persistent hypotension, despite adequate fluid resuscitation, as well as multi-organ dysfunction, diffuse erythroderma and evidence of infection with GAS $[8,9,12]$. These signs and symptoms were observed in the patient.

Early diagnosis is essential given that delayed treatment leads to increased mortality, but it takes difficulties. The diagnosis of necrotizing fasciitis is primarily based on clinical findings. Early local signs are edema, erythema, crepitus, fluctuation, and tenderness, which are nonspecific [1,9]. As the disease progresses, the erythema may darken to a purple hue, bullae may form, and progression to frank necrosis may ensue over hours to days [9]. Suspicion of NF should arise with severe pain disproportionate to these local inflammatory findings and the appearance of signs of systemic toxicity. Surgical exploration and biopsy are the key to confirm necrotizing fasciitis and should not be delayed for imaging $[4,8,9]$. Also, blood cultures are often taken but have limited utility. In some studies positive blood cultures was founded in only $20 \%$ of patients with type I necrotizing fasciitis $[4,18]$. In 
our case, diagnosis of NF was made on clinical grounds. The confirmation of diagnosis was done by surgical exploration, blood culture.

Early aggressive debridement of necrotic tissue under antibiotic cover forms the cornerstone of therapy. Patients should be treated in an intensive care unit, where patient's condition can be monitored closely. In this case, broad spectrum antibiotic therapy was started promptly when the patient was hospitalized. 5 hours after hospitalization, surgery and wound debridement was performed. According to patient's condition deteriorating, next day total hysterectomy was made. All time during observation she received fluid infusion, anticoagulant therapy, vasopressors infusion. Also, several multi-disciplinary consultancies including obstetriciangynaecologists, anaesthesiologists, neurologists, neurosurgeons, clinical pharmacologists, abdominal surgeons and microbiologists were made.

However, this patient's rapid deterioration with septic shock and multisystem organ failure resulted to maternal death. This clinical presentation is not an uncommon scenario treating invasive GAS infections. Davies et al. published study, where the patients mortality rate due to GAS was $15 \%$ overall $(29 \%$ among those over 64 years of age $(P<0.001))$ and $81 \%$ among those with toxic shock $(P<0.001)$ [18]. Moreover, it is observed that death within 7 days due to streptococcal toxic shock syndrome reaches $44 \%$ of all cases [19]. According to this, clinical suspicion must remain high, despite the rarity of the GAS infections and necrotizing fasciitis following GAS after obstetrical operations, as early diagnosis and treatment are critical.

\section{Conclusion}

Despite a low prevalence, it's highly recommended for clinicians to keep close eye and be suspicious of the signs and symptoms of necrotizing fasciitis. Early diagnosis and treatment remains crucial factors in achieving successful results. The gold standard for diagnosis and treatment remains an extensive surgical exploration and debridement under broad spectrum antibiotic therapy.

\section{References}

1. Rimawi BH, Graybill W, Pierce JY, Kohler M, Eriksson EA, et al. (2014) Necrotizing fasciitis and toxic shock syndrome from Clostridium septicum following a term cesarean delivery. Case Rep Obstet Gynecol 1-5.

2. Psoinos CM, Flahive JM, Shaw JJ, Li Y, Ng SC, et al. (2013) Contemporary trends in necrotizing soft tissue infections in the United States. Surgery 153(6): 819-827.

3. Mills MK, Faraklas I, Davis C, Stoddard GJ, Saffle J (2010) Outcomes from treatment of necrotizing soft tissue infections:
Results from the national surgical quality improvement program database. Am J Surg 200: 790-797.

4. Nakayama J, Busse R (2010) An analysis of vulvar necrotizing fasciitis in the unique and ethnically diverse hawaiian population. Hawaii Med J 69(1): 13-16.

5. Saving Mothers' Lives 2006-2008: Briefing on genital tract sepsis. Centre for Maternal and Child Enquiries 2010.

6. Momoh MA, Ezugworie OJ, Ezeigwe HO (2010) Causes and management of puerperal sepsis: The health personnel view point. Advan Biol Res 4(3): 154-158.

7. Smaill FM, Gyte GM (2010) Antibiotic prophylaxis versus no prophylaxis for preventing infection after cesarean section. Cochrane Database Syst Rev 1: CD007482.

8. Mason KL, Aronoff DM (2012) Postpartum group A Streptococcus sepsis and maternal immunology. Am J Reprod Immunol 67(2): 91-100.

9. Roman MA, Laupland KB (2007) Necrotizing fasciitis: Speed is critical to saving life and limb. Parkhurst exchange 15.

10. Sarani B, Strong M, Pascual J, Schwab CW (2009) Necrotizing fasciitis: Current concepts and review of the literature. J Am Coll Surg 208(2): 279-288.

11. Stephenson H, Dotters DJ, Katz V, Droegemueller W (1992) Necrotizing fasciitis of the vulva. Am J Obstet Gynecol 166: 1324-1327.

12. Oud L, Watkins $P$ (2014) Necrotizing fasciitis associated with pregnancy: A population-based cohort Study. Infect Dis Ther Dec 3(2): 307-320.

13. DeMuro JP, Hanna AF, Chalas E, Cunha BA (2012) Polymicrobial abdominal wall necrotizing fasciitis after cesarean section. J Surg Case Rep 9:10.

14. Puvanendran R, Jason Chan Meng Huey, Pasupathy S (2009) Necrotizing fasciitis. Can Fam Physician 55(10): 981-987.

15. Miller LG, Perdreau-Remington F, Rieg G, Mehdi S, Perlroth J, et al. (2005) Necrotizing fasciitis caused by community-associated methicillin-resistant Staphylococcus aureus in Los Angeles. N Engl J Med 352(14): 1445-1453.

16. Deutscher M, Lewis M, Zell ER, Taylor TH, van Beneden C, et al. (2011) Incidence and severity of invasive Streptococcus pneumoniae, Group A Streptococcus, and Group B Streptococcus infections among pregnant and postpartum women. Clin Infect Dis 53(2): 114-123.

17. Lamagni TL, Darenberg J, Luca-Harari B, Siljander T, Efstratiou A, et al. (2008) Epidemiology of severe streptococcus pyogenes disease in Europe. J Clin Microbiol 46(7): 2359-2367.

18. Wong CH, Chang HC, Pasupathy S, Khin LW, Tan JL, et al. (2003) Necrotizing fasciitis: Clinical presentation, microbiology, and determinants of mortality. J Bonr Joint Surg Am 85-A:1454.

19. Davies HD, McGeer A, Schwartz B, Green K, Cann D, et al. (1996) Invasive group A Streptococcal infections in Ontario, Canada. Ontario Group A Streptococcal study group. N Engl J Med 335(8): 547-554. 